\title{
Cellular and organellar membrane-associated proteins in haloarchaea: Perspectives on the physiological significance and biotechnological applications
}

\author{
CAI Lei ${ }^{1}$, ZHAO DaHe ${ }^{1}$, HOU Jing ${ }^{1}$, WU JinHua ${ }^{1}$, CAI ShuangFeng ${ }^{1}$, \\ DASSARMA Priya ${ }^{2} \&$ XIANG Hua ${ }^{1 *}$ \\ ${ }^{1}$ State Key Laboratory of Microbial Resources, Institute of Microbiology, Chinese Academy of Sciences, Beijing 100101, China; \\ ${ }^{2}$ Department of Microbiology \& Immunology, University of Maryland, School of Medicine, Baltimore, MD 21202, USA
}

\begin{abstract}
Halophilic archaea (haloarchaea) inhabit hypersaline environments, tolerating extreme salinity, low oxygen and nutrient availability, and in some cases, high pH (soda lakes) and irradiation (saltern ponds). Membrane-associated proteins of haloarchaea, such as surface layer (S-layer) proteins, transporters, retinal proteins, and internal organellar membrane proteins including intracellular gas vesicle proteins and those associated with polyhydroxyalkanoate (PHA) granules, contribute greatly to their environmental adaptations. This review focuses on these haloarchaeal cellular and organellar membrane-associated proteins, and provides insight into their physiological significance and biotechnological potential.
\end{abstract}

halophilic archaea, membrane proteins, physiology, biotechnology

Citation: Cai L, Zhao D H, Hou J, et al. Cellular and organellar membrane-associated proteins in haloarchaea: Perspectives on the physiological significance and biotechnological applications. Sci China Life Sci, 2012, 55: 404-414, doi: 10.1007/s11427-012-4321-z

Althouth halophilic archaea (or haloarchaea) were isolated from salted fish by the early 1900s [1], they were categorized as a type of bacteria until 1978, when the archaeal domain was first recognized by Carl Woese et al. [2]. Inhabiting hypersaline environments, halophilic archaea tolerate high osmotic pressure and require molar salt concentrations (generally above $2 \mathrm{~mol} \mathrm{~L}{ }^{-1} \mathrm{NaCl}$ ) to ensure cellular integrity and active growth [3].

Facing the challenges of the high salt concentrations and other extreme conditions, haloarchaea have evolved several quite unique membrane-associated proteins. The S-layer proteins, located at the cell surface, have been suggested to be important for maintaining cell structure in halophilic archaea in order to adapt to harsh environments [4]. A variety of transporters, distributed in the cellular membrane, are

*Corresponding author (email: xiangh@im.ac.cn) responsible for the uptake of essential nutrients, excretion of deleterious substances, and are important for haloarchaea to selectively import ions into the cytoplasm to maintain internal osmolarity [5,6]. Notably, the twin-arginine translocation (Tat) pathway, but not the general secretion (Sec) pathway, plays a dominant role in protein transport in halophilic archaea [7,8]. Significantly, some haloarchaea have a group of specific retinal proteins in the membrane, including bacteriorhodopsin (a proton pump), halorhodopsin (a chloride pump) and sensory rhodopsins (phototaxis receptors), which are important for their adaptation to microaerobic conditions, high salinity and intense radiation environments [9-12]. In addition to those functioning in and associated with the cellular membrane, intracellular proteins making up the membranes of gas vesicles [13] and located on PHA granules [14-17] are also very important for the 
physiological adaptation of haloarchaea.

As a result of in-depth research using genetic, metabolic and biochemical tools, as well as genomic approaches, a better understanding of the various haloarchaeal membrane-associated proteins, from structure to physiological mechanisms, has been achieved. Moreover, an increase in the number of biotechnological applications using these unique proteins can be expected in the near future. Here, we provide a brief review of the known haloarchaeal cellular and organellar membrane-associated proteins, and focus on their physiological significance and biotechnological potential.

\section{S-layer proteins}

Regularly arrayed S-layer proteins are a common feature of the cell envelopes of almost all archaea [18] and some bacteria. These protein arrays are composed of oligomeric units which are anchored in the plasma membrane, with extended spacers maintaining the bulk of the porous surface layers at a constant distance above the membrane surface $[18,19]$. The first archaeal S-layer in haloarchaea was identified in 1956, through the analysis of changes in cell shape with the antibiotic bacitracin [20]. Different from the phospholipid bilayers found in bacteria or eukaryotes, archaeal plasma membranes contain tetraether lipids [21]. S-layers which are expected to maintain or determine the cell shape represent the sole cell wall component in most of the archaea [4]. In the mid 1970s, with the chemical characterization of S-layer proteins of haloarchaea [22], archaea, especially the halophilic archaea, became an important research focus for the study of S-layer proteins [23-26]. The haloarchaeal S-layer proteins are rich in acidic residues. The dominance of acidic residues may facilitate the correct folding and functioning of haloarchaeal proteins under high-salt conditions [27-29]. In addition, the glycosylation of S-layers was also discovered in the 1970s [22,30].

While S-layer (glyco)proteins have been identified in archaea living in a variety of extreme conditions, the best characterized S-layer glycoproteins are those from halophilic archaea. Three haloarchaeal S-layer glycoproteins have been studied in detail, i.e., the S-layer glycoproteins of a Halobacterium strain [26], Haloferax volcanii [25] and Haloarcula japonica [24]. The S-layer protein from $H$. japonica shows $52.1 \%$ and $43.2 \%$ identity to those from $\mathrm{Ha}$ lobacterium and $H$. volcanii, respectively [24]. The mature S-layer protein of $H$. japonica contains 828 amino acids, with a single, highly hydrophobic stretch at the C-terminus [24]. This C-terminal hydrophobic region probably serves as a membrane anchor, and this motif has also been observed in the S-layer protein of Halobacterium [26]. Both $\mathrm{N}$ - and O-glycosylations have been discovered in S-layer glycoproteins [25,26]. The $\mathrm{N}$-glycosylation sites are scattered throughout the sequence; however, O-glycosylation sites are usually found clustered in a threonine-rich region. At least two of the seven putative $\mathrm{N}$-glycosylation sites of the S-layer glycoprotein in the haloarchaeaon $H$. volcanii are modified by a pentasaccharide, which comprises a hexose, two hexuronic acids, a methyl ester of hexuronic acid and a mannose [31]. Five glycosyltransferases named AglJ, AglG, AglI, AglE and AglD are sequentially involved in the transfer of five residues to the dolichol phosphate carrier directly or indirectly [32-35]. Finally, the oligosaccharyltransferase AglB delivers the pentasaccharide to S-layer glycoprotein Asn residues [32,34].

Interestingly, though only neutral oligosaccharides have been discovered in the S-layer glycoprotein of the moderate halophile $H$. volcanii (with optimal growth at 1.7-2.5 mol $\mathrm{L}^{-1} \mathrm{NaCl}$ ) [36] and the S-layer glycoproteins of the extreme halophile Halobacterium (with optimal growth at 3-5 mol $\mathrm{L}^{-1} \mathrm{NaCl}$ ) [12] contain sulfated glucuronic acid residues and a higher degree of glycosylation. These differences in glycosylation are thought to be mainly caused by the different salinities found in their environments, and not because of the difference of S-layer proteins [37]. As these differences lead to an increased density in surface charges in Halobacterium, it renders the cells better adapted to the higher salt concentrations [36].

\section{Channels and transporters}

Channels and transporters in the membranes of haloarchaea are involved in maintenance of the high intracellular osmotic pressure to ensure cellular integrity, and transportation of nutrients for active metabolism, and therefore are important for halophilic archaea to live in the hypersaline environments.

In the halophilic archaeon $H$. volcanii, two types of mechanosensitive (MS) channels have been identified, named MscA1 and MscA2 (MS channels of archaea). The major difference between archaeal- and bacterial-type MS channels lies in their sensitivities to pressure, which are 1.4-2.9 $\mathrm{mmHg}$ per e-fold change in open probability of channels for the archaeal-type [38] and approximately 5 $\mathrm{mmHg}$ per e-fold change for the bacterial-type [39]. MscA1 and MscA2 differ from each other in their distinct rectification properties: MscA1 has a conductance of $380 \mathrm{pS}$ (pressure sensitivity) at $140 \mathrm{mV}$ and $680 \mathrm{pS}$ at $240 \mathrm{mV}$, whereas MscA2 has a conductance of $850 \mathrm{pS}$ at $140 \mathrm{mV}$ and $490 \mathrm{pS}$ at $240 \mathrm{mV}$ [38]. Interestingly, these archaeal type MS channel proteins still preserve their bioactivities when dissolved by detergents and reconstituted into liposomes in patch-clamp experiments [38,39].

The research on primary transporters of archaea has focused primarily on the ABC family of transporters, which constitute one of the largest protein families in existence [40]. The classical archaeal- and bacterial-type ABC transporters have the same architecture, and are composed of a 
substrate-binding protein on the outside of the membrane, two integral membrane permeases and two ATPases located at the cytoplasmic membrane [41].

The ABC transporters are essential for the viability of halophilic archaea. Three ABC transporters of $H$. volcanii have been reported, which are glucose specific, molybdate specific and anion specific [42]. Recent studies on Halobacterium have revealed the existence of a putative $\mathrm{ABC}$ transporter for corrinoid use in haloarchaea [43]. Currently, the best studied ABC transporters in archaea have been those related to sugar uptake. Alignment analysis of seven archaeal ABC transporter MalK subunits (including Haloarcula marismortui), which are the motor units of the trehalose/maltose system, showed many conserved amino acid sequences, including the Walker $\mathrm{A}, \mathrm{B}$ and $\mathrm{H}$ motifs, the Q-loop or linker region, the ABC signature (LSGGQ), the regulatory domain motif (RDM) GIRPE and phenylalanine (F) at the C-terminus [44].

There are some other transporters which are driven by electrochemical gradients, and three modes (uniporter, symporter and antiporter), that can be deduced from the genome sequence of the halophilic archaea. Uniporters are integral membrane proteins that facilitate diffusion, translocating only one solute that equilibrates along its electrochemical gradient, e.g., the uptake of phosphate relies on three uniporter proteins, PitA1, PitA2 and PitA3 in Haloquadratum walsbyi [45]. Symporters usually co-transport a solute with an ion, e.g., the sodium/sulfate and sodium/proline symporters are considered to be based on the proteins CitT and PutP in Haloferax mediterranei (unpublished data). Antiporters exchange solute for an ion or another substrate, e.g., the $\mathrm{Na}^{+} / \mathrm{H}^{+}$antiporter (NhaC) in $\mathrm{Ha}$ lobacterium species NRC-1 [29].

The sugar-phosphotransferase system (PTS) transports sugars into cells using phosphoenolpyruvate (PEP) as the phosphoryl donor to phosphorylate sugars, and is commonly found in bacteria. After analysis of all 16 completely sequenced haloarchaeal genomes, we found that nine haloarchaea have PTS homologues (Figure 1). H. mediterranei, $H$. marismortui, H. volcanii, Haloarcula hispanica, Halakalicoccus jeotgali and Haloterrigena turkmenica each code for five PTS-related proteins, including PtsC, PtsA, Hpr, PtsI and PtsB, which constitute a complete PTS system, but the other three, H. walsbyi, Halorubrum lacusprofundi and Haladaptatus paucihalophilus only code for Hpr and PtsI. PtsI autophosphorylates from PEP and transfers a phosphoryl group to Hpr, and PtsA becomes phosphorylated by the phosphorylated-Hpr and passes the phosphoryl group on to PtsB. PtsC functions as a permease and sugar-specific receptor located in the cell membrane [46]. PtsA, PtsB and PtsC are components of an enzyme complex named enzyme II. The lack of the enzyme II complex in these three halophiles indicates that they either possess an alternate mechanism, or that they cannot transport sugars across the cell membrane via the PTS system but instead contribute to the phosphoryl transmission in sugar of phosphorylation. Examination of the genomes of thermophiles identified PTS system genes only in the hyperthermophile, Thermofilum pendens [47]. Further analysis of genome sequences of all the published 109 archaea did not reveal any other cases (unpublished data).

\section{Protein transportation systems}

There are two major pathways for protein transportation across the cytoplasmic membrane. One is the general secretion route (Sec pathway), which catalyzes the transmembrane translocation of proteins in their unfolded conformation, and the other one is the Twin-arginine translocation pathway (Tat pathway), which catalyzes the secretion of folded and/or cofactor containing proteins [7,8]. Protein translocation mainly depends on the Sec pathway in all three domains, except in haloarchaea, where protein translocation is achieved mainly by the Tat pathway [48,49], indicating that they prefer to translocate folded proteins across the cytoplasmic membrane.

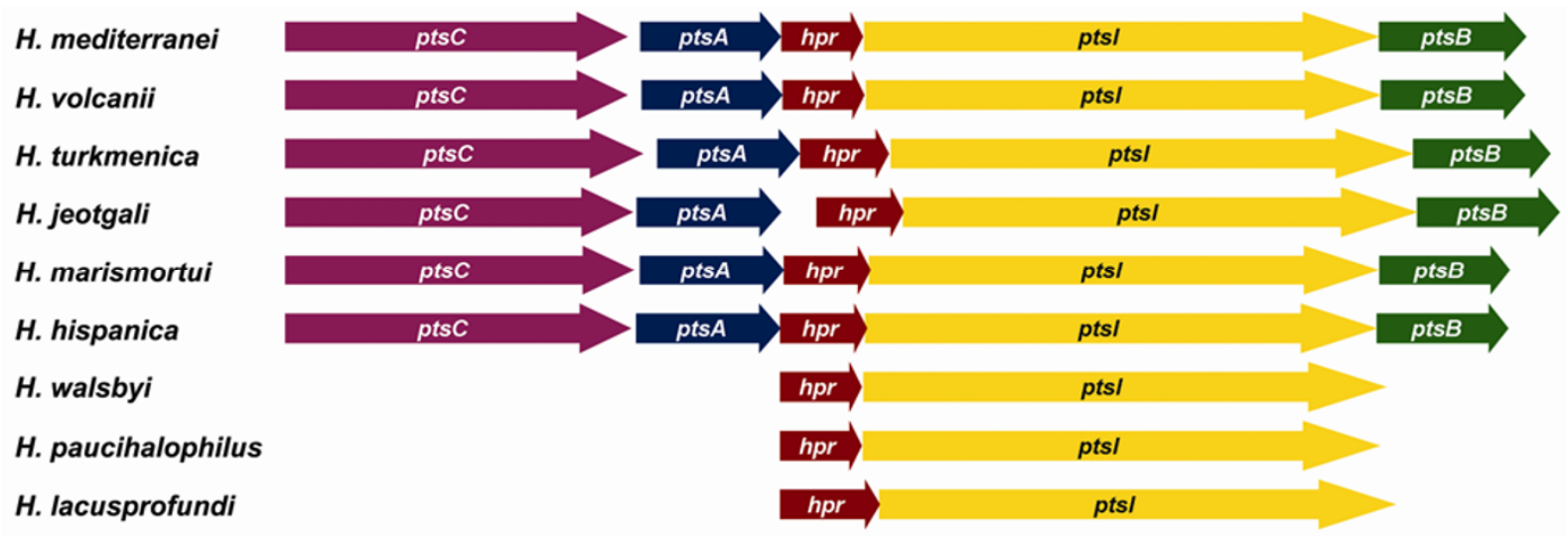

Figure 1 Schematic representation of the gene clusters encoding the PTS system in nine halophilic archaea, with six that have the complete PTS system containing the enzyme I (Hpr and PtsI) and enzyme II (PtsA, PtsB, and PtsC). 
Although the energy of the Tat pathway comes solely from the proton motive force (PMF), the Tat pathway substrates include not only secreted soluble proteins, but also C-terminally anchored proteins and lipoproteins [50,51]. These proteins make up more than half of the number of putative secretion proteins $(>60 \%)$ in halophilic archaea [48]. The TATFIND software and a facile reporter system were used to predict the Tat substrates and identify Tat signal peptides from all three domains of life $[52,53]$. The Tat substrate signal peptides were found to contain a consensus motif: (S/T)RRxФФ (where $\mathrm{S}$ is a serine, $\mathrm{T}$ is a threonine, $\mathrm{R}$ is an arginine, $\mathrm{x}$ is any residue and $\Phi$ is a hydrophobic residue), where the twin-arginine is invariant [54].

In halophilic archaea, two genes (tatA and tatC) encode the core complex (TatAC) of the Tat system [55], whereas in bacteria and eukarya, the Tat system is usually composed of three components, including TatA (Tha4 and Tha9 in the plant chloroplast system), TatB (Hcf106 in plants) and TatC [56]. Each halophilic archaeon encodes one or two tatA and two tatC homologues generally named tatAo, tatAt, tatCo and tatCt, and the last three genes are essential for viability [55,57]. TatAo and TatAt each have an N-terminal hydrophobic transmembrane segment (TMS) followed by one or two C-terminal cytoplasmic helices [7,55]. A typical TatC protein termed TatCo has six TMSs with a long cytosolic N-terminus, whereas the TatCt protein has $14 \mathrm{TMSs}$, suggesting that two molecules of TatCo are connected by two additional TMSs [8,55]. The mechanism of the Tat pathway is unclear, and many hypotheses have been put forward on the basis of different experimental results [56]. In $E$. coli, the TatA protein can insert into the membrane and is presumed to form a channel for Tat substrate translocation; however, in B. subtilis, TatA is soluble in the cytoplasm. Based on the above observations, more in-depth studies on the three dimensional structure of the Tat complex is necessary.

\section{Retinal proteins}

At least four retinal-containing membrane proteins have been identified in halophilic archaea: bacteriorhodopsin (BR), halorhodopsin (HR), and the sensory rhodopsins I and II (SRI and SRII) (Figure 2). They are also termed archaeal rhodopsins because of their overall structure, photoreactions and use of retinal chromophore as in visual rhodopsins [59]. All four proteins share a common structure of seven transmembrane helices (A, B, C, D, E, F and G), with a retinal chromophore binding through a protonated Schiff base to the lysine residue in the seventh helix $(G)$.

BR was first discovered in halophilic archaea in the 1970s [60]. It is the only protein found in the purple membrane (PM) of Halobacterium, induced when grown under low oxygen and high light intensity conditions. PM contains $75 \%$ BR protein and $25 \%$ lipid, in which BR exists in the form of trimers that assemble into a $2 \mathrm{D}$ hexagonal crystal lattice in the PM $[61,62]$. BR is encoded by the bop gene and contains a single polypeptide chain of 248 amino acids, with the retinal chromophore bound to the $\varepsilon$-amino group of Lys-216 [63-65]. When treated with detergents, the BR monomers can be completely isolated from the lipids $[63,66]$.

Physiologically, BR functions as a light-driven proton pump, whose mechanism has been extensively studied. Normally, BR exists either in a light-adapted or a dark-adapted form. The light-adapted form contains only all-trans retinal whereas the dark-adapted form contains equal amounts of 13-cis and all-trans retinal. Both forms go through a cyclic photoreaction, but only the all-trans retinal-containing BR translocates protons across the membrane $[67,68]$. Briefly, proton translocation by BR can be described as follows. First BR $_{570}$ absorbs photons, causing the all-trans retinal to be isomerized into the 13-cis configuration, forming five intermediates in turn: $\mathrm{K}, \mathrm{L}, \mathrm{M}, \mathrm{N}$, and

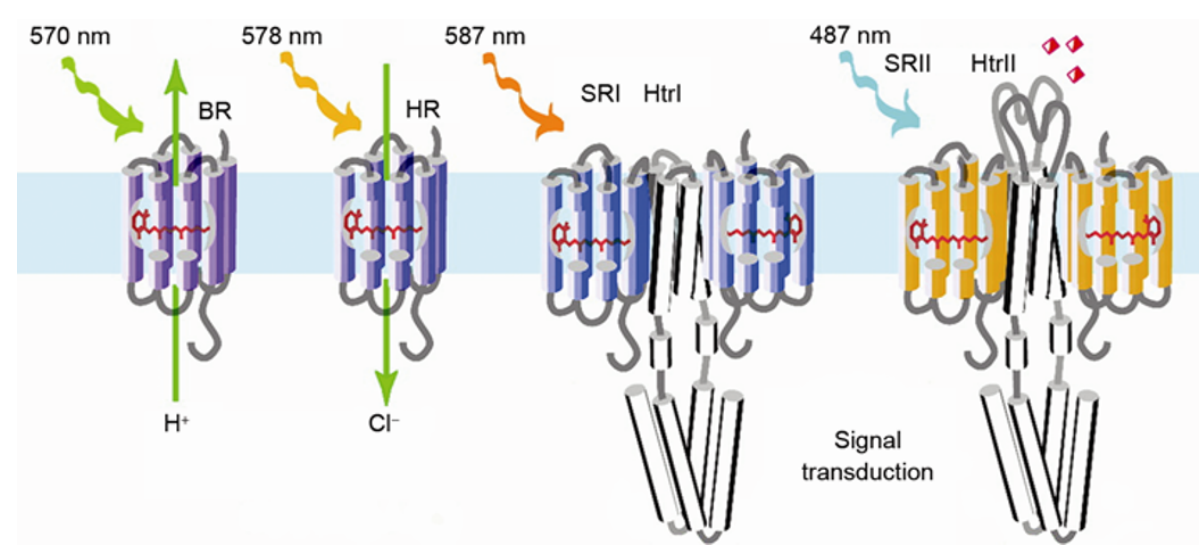

Figure 2 The four archaeal rhodopsins in Halobacterium. The proton pump bacteriorhodopsin (BR), the chloride pump halorhodopsin (HR), phototaxis receptors sensory rhodopsins I and II (SRI and SRII) are shown. All four proteins share a common structure of seven transmembrane helices with a retinal chromophore binding through a protonated Schiff base to the lysine residue in the seventh helix. Unlike BR and HR, the sensory rhodopsins form complexes with their transducer proteins HtrI and HtrII, which modulate kinase activity, that in turn controls the flagellar motor (modified from Spudich et al. [58]). 
$\mathrm{O}$, which differ in the states of the retinal and their surrounding environment [10,69-71]. In the end, BR returns to its initial state $\left(\mathrm{BR}_{570}\right)$ and a proton is transferred from the cytoplasmic side to the extracellular surface of the membrane, generating an electrochemical gradient across the membrane [63,72,73], which consequently drives other metabolic processes, e.g., ATP synthesis and active transport. The $\mathrm{M}$ state plays a key role in this cycle, as its formation coincides with the pumping of protons [72]. Conformational change of BR from one state to another can be as fast as a picosecond and the cycle can be completed in $\sim 10$ ms $[69,74]$.

Halorhodopsin (HR) is the second retinal protein and is ubiquitous in halophilic archaea. It is encoded by the haloopsin (hop) gene and has a molecular weight of $\sim 20 \mathrm{kD}$. HR shares a similar structure with BR, except that in HR the chromophore binding site is Lys-242. HR was first found to act as an inward-oriented light-driven chloride pump [75]; however, later studies revealed that it could also pump other anions like bromide, iodide and nitrate [11]. HR is essential for maintaining high internal chloride concentrations, as high concentrations of intracellular $\mathrm{KCl}$ are the general strategy used by haloarchaea to maintain osmotic balance during cell growth, and light-driven anion pumps can considerably reduce the use of metabolic energy. Similar to BR, HR also has two different forms: a dark-adapted form and a light-adapted form, but in this case, both forms contain a mixture of all-trans and 13-cis retinal chromophores. It is known that HR goes through a six-step photocycle, in which the photo intermediates are $\mathrm{HR}_{578}, \mathrm{HR}_{600}, \mathrm{HR}_{520}, \mathrm{HR}_{640}$, and $\mathrm{HR}_{625}$ $[9,76,77]$. What is different is that HR lacks the long-lifetime $\mathrm{M}$ intermediate, which is characteristic of a deprontonated Schiff base. The functional role of HR differs under different light conditions. Green light causes chloride transport whereas additional blue light induces HR to pump protons in an opposite direction to $\mathrm{BR}$ [76]. Halorhodopsin from the haloalkaliphilic archaeon Natronomonas pharaonis behaves quite differently. It acts more like an outward facing proton pump in the presence of azide [9]. BR can also be converted to an HR-like pump when it binds to halide ions [78].

Two sensory rhodopsins (SR), named SRI (or slowcycling sensory rhodopsin) and SRII (or phoborhodopsin), exist in halophilic archaea. SRI was first described in 1982 as a third rhodopsin-like pigment in Halobacterium $[79,80]$. Later in 1986, SRII was identified using Flash spectrophotometric methods. Both sensory rhodopsins I and II are structurally similar to BR, except that they form complexes with two haloarchaeal proteins, HtrI and HtrII, respectively. These two proteins serve as transducers in the complex. Each of them has two transmembrane helices (TM1 and TM2) and two conserved regions: the signaling domain and the stimulus relay domain [80-82]. Previous studies revealed that both SRs were responsible for color-sensitive phototaxis signaling in halophilic archaea using a similar mechanism, with the difference being that SRI mediates both attractant and repellent responses, whereas SRII mediates a repellent response only [81]. The process can be briefly described as follows: when a change in color and light intensity occurs, SRI and SRII absorb photons, which leads to the photoisomerization of retinal from all-trans to 13-cis $[81,83]$. Then the SR-Htr complexes undergo extensive structural changes that further modulate cytoplasmic phosphorylation cascade reactions and in turn control the reversal frequency of the flagellar motor in response [84-86]. When isolated from their complex regulator proteins, SRs can pump protons just like BR [79].

\section{Gas vesicles}

Intracellular gas-filled vesicles (GV, Figure 3) have been identified in several Haloarchaea, including Halobacterium species NRC-1, H. mediterranei, Halogeometricum borinquense and $H$. walsbyi. They are phase- bright, low-density, buoyancy organelles, made of an extremely stable, rigid, gas-permeable, lipid-free, protein membrane. Water is excluded from the interior as a consequence of the hydrophobicity of the interior surface. They regulate buoyancy in some bacterial and archaeal cells, allowing them to float closer to light and oxygen near the surface. Gas vesicles vary widely in size, from $50 \mathrm{~nm}$ to over $1 \mu \mathrm{m}$ in length, and $30-250 \mathrm{~nm}$ in width. Those haloarchaea found at lower depths, and exposed to higher hydrostatic pressures, are generally found to have gas vesicles which are narrower, possessing a long cylindrical midsection and conical end caps, whereas species found in shallower environments and experiencing lower pressures have wider, more lemon-shaped gas vesicles [87].

Though easily purified by lysis of cells followed by floatation, gas vesicles are extremely resistant to solubilization, and this has made biochemical identification of the exact proteins found in the membrane difficult. Initially, they were thought to consist solely of a self-assembling protein, GvpA, because of it being the most abundant. However, the biogenesis is more complex based on the
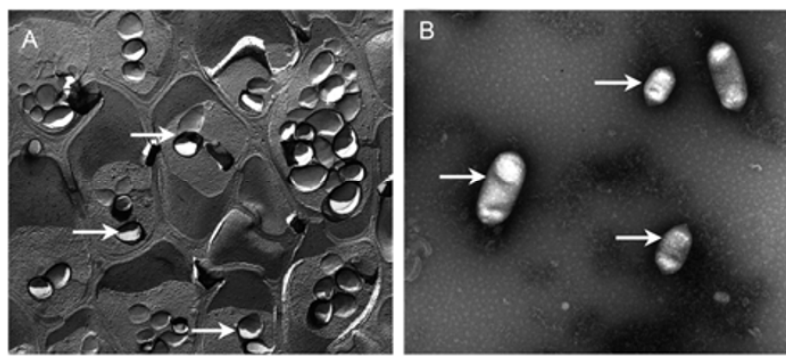

Figure 3 Gas vesicles of haloarchaea. A, Freeze fracture electron micrograph of Halobacterium sp. NRC-1 cells. Outlines of cells are visible and where the fracture plane has broken through cells, gas vesicles are visible (indicated by arrows). B, Electron micrograph of isolated gas vesicles from Haloferax mediterranei. 
number of genes implicated in halophilic archaea, many of which code for minor structural, assembly and regulatory proteins. In haloarchaea, gas vesicle genes are found both on plasmids and on chromosomes and are often organized in clusters. In Halobacterium, the gvp genes are organized in a large gene cluster on unstable megaplasmids [13]. The 14 genes are organized into a divergently organized cluster: $g v p A, C, N$ and $O$ in the rightward operon and $g v p D, E, F$, $G, H, I, J, K, L$ and $M$ in the leftward operon. In Halobacterium, genetic analysis has identified those required for wildtype gas vesicle synthesis $[88,89]$. In electron micrographs, the membrane appears as a ribbed structure. Within the cylindrical portion, the ribs form a shallow right-handed spiral starting from the cones and meeting in the central region, where growth is thought to occur by the addition of protein subunits. GvpA is predicted to adopt a coil- $\alpha-\beta-\beta-\alpha$-coil structure, where the antiparallel $\beta$-strands form a hydrophobic surface [90,91]. A second gas vesicle protein, GvpC, binds to the outside of the vesicle and helps to strengthen the structure against collapse [92]. It possesses seven internally-homologous repeats of 25 to 38 amino acid residues in addition to an acidic tail at the C-terminus [93-95]. Each repeat that constitutes the $\alpha$-helical structure is long enough to span a rib formed by GvpA, raising speculation that each repeat of GvpC acts as a clamp to connect two ribs [96]. The predicted GvpN protein has an AAA-family ATPase motif, consistent with an energy requiring function. Absence of the GvpC or GvpN results in the production of extremely small gas vesicles, whereas others appear necessary for proper shape and stability. The $\operatorname{svp} O, D$ and $E$ genes are thought to be involved in the regulation of gas vesicle gene expression, whereas the $g v p J$ and $M$ genes encode minor proteins similar to GvpA, and $g v p F$ and $L$ specify minor proteins with coiled-coil domains, usually involved in self-association [97]. Recently, the GvpA, C, F,

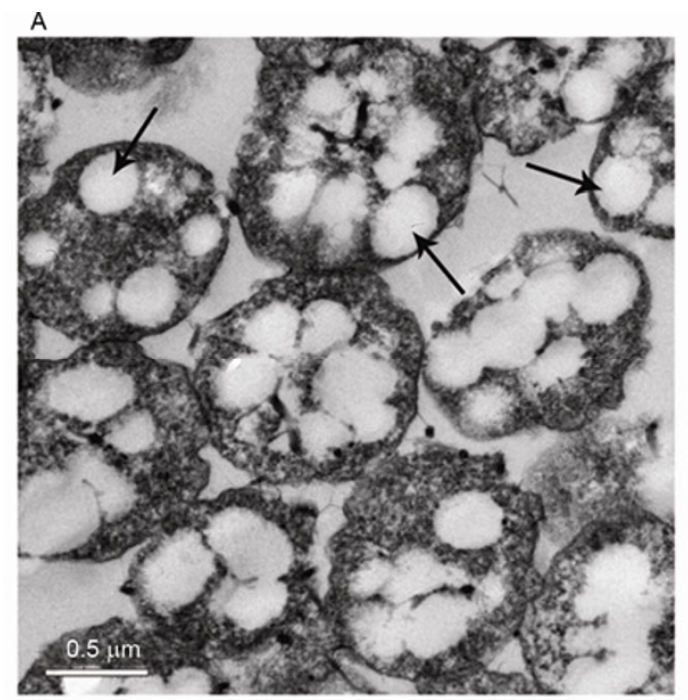

G, I, J, L, M and N have been identified by proteomic analysis of purified gas vesicles from Halobacterium [98].

\section{Polyhydroxyalkanoate (PHA) granule associ- ated proteins}

With an oversupply of carbon sources, many haloarchaeal strains in the genera Haloferax, Halobacterium, Haloarcula and Haloquadratum can synthesize PHAs, which are accumulated intracellularly in the form of inclusion bodies (PHA granules). Some of these halophilic archaea, including Halopiger aswanensis sp. nov. [99] and Haloferax mediterranei ATCC 33500, are able to accumulate PHAs up to $\sim 60 \%$ of their dry weight $[100,101]$. It has been revealed that a number of proteins are located on the surface of PHA granules and are essential for PHA synthesis in bacteria, namely, PHA synthases, depolymerases, regulatory proteins and phasins. In halophilic archaea, studies on these PHA granule associated proteins (PGAPs) are sparse, and a few PGAPs have recently been discovered by the Xiang laboratory (Figure 4) [14-16].

The first PGAP in haloarchaea was reported in 2007 [16] when the PHA synthase in $H$. marismortui was identified. PHA synthase is the key enzyme of PHA synthesis and catalyzes the polymerization of the $(R)$-3-hydroxyacyl-CoA monomers to PHA. Currently, PHA synthases have been divided into four classes depending on their primary structures, subunit compositions and substrate specificity in bacteria [102]. The haloarchaeal PHA synthases investigated seem to be more similar to the bacterial class III synthase, containing PhaE and PhaC subunits. However, the PhaC subunits of halophilic archaea are much larger and contain a longer $\mathrm{C}$ terminus than their bacterial counterparts,

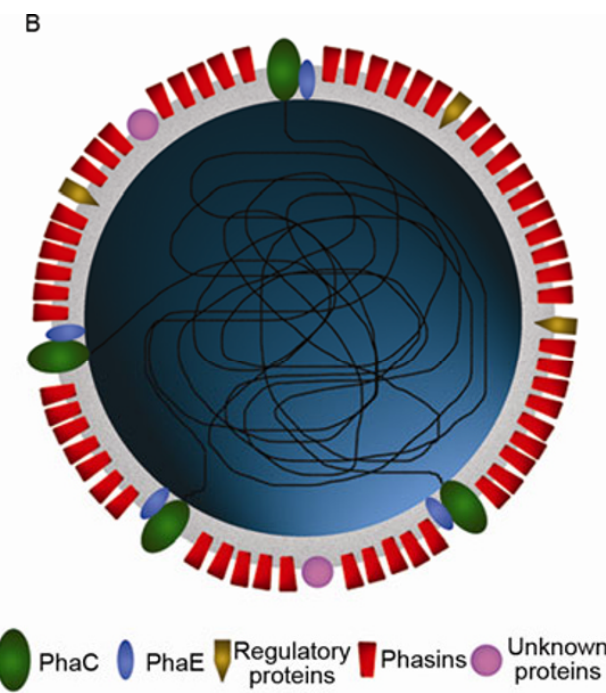

Figure 4 Electron micrographs of ultrathin sections of PHA granules indicated by arrows in H. mediterranei (A) and a schematic depiction illustrating various proteins located at the haloarchaeal PHA granule surface (B). 
and the haloarchaeal PhaE subunits $(\sim 20 \mathrm{kD})$ are much smaller than those of bacteria $(\sim 40 \mathrm{kD})$. Western blotting analysis revealed that both $\mathrm{PhaC}$ and $\mathrm{PhaE}$ are bound to the PHA granules in $H$. mediterranei, whereas in $H$. marismortui, only PhaC has been demonstrated to be strongly bound to the PHA granules $[15,16]$.

While the PHA synthases of haloarchaea have shown some homology to the bacterial synthases, the other PGAPs, i.e., the depolymerase PhaZ, regulator PhaR and the phasin PhaP, found in bacteria, have not been identified in any haloarchaeal genome, indicating that they may be quite distinct from their bacterial counterparts. In 2012, the predominant structure protein (phasin) on the PHA granules, PhaP, was identified by two-dimensional gel electrophoresis and MALDI TOF/TOF MS in $H$. mediterranei and showed a significant effect on PHA accumulation and PHA granule formation. In addition, bioinformatic analysis indicated that this kind of PhaP is conserved only in haloarchaea and represents a haloarchaeal-type PHA phasin [14].

In bacteria, the regulatory protein (PhaR) tightly regulates PHA granule formation and phasin production. When PHAs are synthesized, PhaR could bind to the hydrophobic granule surface, reducing the cytoplasmic concentration of PhaR to a low level, which enables the transcription of phaP. Once PhaP is synthesized, it binds to PHA granules immediately; when PHA granules reach their maximum size, most of the granule surface is found to be covered with PhaP proteins, leaving no binding site for PhaR. Increasing cytoplasmic concentrations of PhaR represses transcription of phaP and its own gene, indicating an efficient autoregulation of phaR expression [103-105]. Although PhaR has not been reported in haloarchaea, a recent 2D-gel analysis of PHA-associated proteins in $H$. mediterranei has revealed a protein with a DNA-binding motif (unpublished data), which may represent the regulatory protein in the archaeal domain.

\section{Biotechnological potentials}

With the study of haloarchaeal cellular and organellar membrane-associated proteins continuing, the biotechnological potential of these proteins have also emerged. Since haloarchaeal cells are easily lysed, therefore leading to the release of intracellular particles and membrane components, their cellular components are readily produced for many novel applications [106].

S-layer proteins are capable of self-assembly into monomolecular crystalline arrays in suspension, or on suitable surfaces and interfaces, and are more easily harvested than their bacterial counterparts. Hence, as a basic building block, S-layer proteins are suited for nanobiotechnological applications in the construction of all major classes of biological molecules (proteins, lipids, glycans, nucleic acids and combinations thereof) [107-110]. In addition, the halo- archaeal cell envelope, composed of a cytoplasmic membrane and a closely associated monomolecular crystalline (glyco)protein lattice, is stable under high salt concentrations, which has led to plans to exploit this supramolecular principle for stabilizing planar or vesicular membrane systems [111].

The reason that rhodopsins in halophilic archaea have attracted so much attention in the last three decades is that they hold significant potential for applications in the field of biotechnology. BR possesses an extremely stable hexagonal lattice structure, and is resistant against chemical, thermal and photochemical degradation [68], which makes it a potentially optimal biomaterial for use in biological devices under conditions of high temperature, strong light intensity and high ionic strength. More importantly, the optical properties of BR, and its ability to form two-dimensional films are being investigated for use in optical nanodevices, including exploitation of the photochromic properties of BR, which mainly involves interchange of the initial B and M states. This can be used for high-capacity memory storage, including spatial light modulators, holographic associative memories, 2-D and 3-D data storage, volumetric memory, and protein-based associative processors and may be suitable for use in most molecular electronic devices [10,84,112-114]. Another important characteristic of BR, its nonlinear property, can be applied in devices such as gated multipliers and optical amplifiers. Furthermore, the photoelectronic aspect of bacteriorhodopsin, i.e., lightdriven proton translocation and the high speed of the photocycles $(\sim 10 \mathrm{~ms})$, also make retinal proteins outstanding candidates for photo- and bio-sensors [115], nonlinear optical filtering and light switching [84]. BR may also be used for seawater desalination [10]. It is also a candidate for use in gene therapy in neuroscience and has recently attracted tremendous interest [116-118]. Heterogeneously expressed halorhodopsins have been used to restore night vision in mice and potentially could be used as artificial retinas.

The gas vesicles of haloarchaea are highly stable, non-toxic and easily produced on a large scale [119]. Directed expression of the haloarchaeal $g v p$ gene cluster in $E$. coli resulted in gas vesicle biosynthesis in the host and was proposed as a novel way to genetically engineer flotation of cells. In addition, they are easily purified from haloarchaea by lysing cells followed by purification with flotation. Genetic fusions of GvpC with foreign proteins have been constructed for novel biotechnological applications, such as antigen display, which is facilitated by their stability and immunogenicity. Their use as potential vaccine delivery systems has been explored as recombinant gas vesicles, in which the GvpC has been coupled with a hapten or genetically recombined with a model six amino acid peptide to render the gas vesicles immunogenic, eliciting strong specific humoral responses in the absence of exogenous adjuvant. The long-lived responses exhibit immunologic memory, indicative of a natural and slow release of epitopes 
over time, so recombinant gas vesicles can be regarded as a new kind of self-adjuvating display vehicles of peptidyl epitopes [120]. The cassette-based display/delivery system is able to express and deliver peptides of SIV, HIV or other pathogens through DNA recombinant technology, and such a system can be used to construct a multiepitope display (MED) library [121]. In addition to serving as a vaccine display/delivery system, recombinant gas vesicles can also be used as an initial screen of peptides expressed during pathogen infections in vivo [122].

PHAs are considered potential substitutes for petrochemical-derived plastics because of their biodegradable, biocompatible-and thermoplastic features, and can be used as packaging and biomedical materials, in the manufacture of nonwoven fabrics, as well as used for slow release drug delivery [123-126]. Several haloarchaeal strains can synthesize PHAs from cheap carbon sources and isolation of PHAs from these halophilic archaea is much easier when compared with the isolation from bacteria [100]. Beside the application of PHA itself, the potentials of PHA granule proteins in biotechnology is also emerging. PHA synthases immobilizing $\beta$-galactosidase are stable under various storage conditions for several months, which shows that the production of functionalized PHA granules through protein engineering could be a useful tool for developing biological nano-/micro-beads for various applications [127]. In another recent work, the PHA synthase, fusing the IgG-binding domain of protein A to the PhaC N-terminus, has been engineered for the purification of immunoglobulin $\mathrm{G}$ (IgG) [128]. For specific binding and elution of their antigen $\beta$-galactosidase, an anti- $\beta$-galactosidase $\mathrm{scFv}$ (single-chain variable fragment of an antibody) was immobilized at the surface of PHA granules [129]. Streptavidin was fused to the PHA synthase at the C-terminus, which demonstrates that the PHA granules generated in vivo with streptavidin are applicable for ELISA, DNA purification, enzyme immobilization and flow cytometry [130]. Fused to genetically engineered proteins for inorganics (GEPIs), the granules could also serve as suitable tools for medical bioimaging procedures [131]. In addition, PHA granules and their surface-associated proteins have become an ideal material for protein immobilization and purification [132-134]. For instance, when the protein of interest is fused to the C terminus of PhaP, the protein could bind to the granules via the phasin tag. With cell disruption, simple centrifugation and suitable cleavage, the protein can then be separated easily from the granules and phasin tags [135]. Since the PHA granules are much easier to purify from halophilic archaea, the biotechnology of PHA granules-associated proteins may well blossom in the near future.

This work was partially supported by the National High Technology Research and Development Program of China (Grant No. 2010AA09Z401), National Natural Science Foundation of China (Grant Nos. 30621005, 30830004, and 30925001) and Chinese Academy of Sciences (KSCXZ-
EW-G-2-4). P. DasSarma was supported by the US National Aeronautics and Space Administration (Grant No. NNX10AP47G awarded to S. DasSarma).

1 DasSarma P, Klebahn G, Klebahn H. Translation of Henrich Klebahn's 'Damaging agents of the klippfish - a contribution to the knowledge of the salt-loving organisms'. Saline Systems, 2010, 6: 7

2 Woese C R, Magrum L J, Fox G E. Archaebacteria. J Mol Evol, 1978, 11: 245-251

3 Soppa J. From genomes to function: haloarchaea as model organisms. Microbiol-Sgm, 2006, 152: 585-590

4 Messner P, Sleytr U B. Crystalline bacterial cell-surface layers. Adv Microb Physiol, 1992, 33: 213-275

5 Oren A. Bioenergetic aspects of halophilism. Microbiol Mol Biol Rev, 1999, 63: 334-348

6 Albers S V, Van de Vossenberg J L, Driessen A J, et al. Bioenergetics and solute uptake under extreme conditions. Extremophiles, 2001, 5: 285-294

7 Albers S V, Szabó Z, Driessen A J M. Protein secretion in the Archaea: multiple paths towards a unique cell surface. Nat Rev Microbiol, 2006, 4: 537-547

8 Natale P, Brüser T, Driessen A J M. Sec- and Tat-mediated protein secretion across the bacterial cytoplasmic membrane Distinct translocases and mechanisms. BBA-Biomembranes, 2008, 1778: 17351756

9 Schäfer G, Engelhard M, Müller V. Bioenergetics of the archaea. Microbiol Mol Biol R, 1999, 63: 570-620

10 Hampp N. Bacteriorhodopsin as a photochromic retinal protein for optical memories. Chem Rev, 2000, 100: 1755-1776

11 Lanyi J K. Halorhodopsin: a light-driven chloride ion pump. Annu Rev Biophys Biophys Chem, 1986, 15: 11-28

12 DasSarma S, DasSarma P. Halophiles. In: Encyclopedia of Life Sciences. Chichester: John Wiley \& Sons, 2012

13 DasSarma S, Arora P. Genetic analysis of the gas vesicle gene cluster in haloarchaea. Fems Microbiol Lett, 1997, 153: 1-10

14 Cai S, Cai L, Liu H, et al. Identification of the haloarchaeal phasin (PhaP) that functions in polyhydroxyalkanoate accumulation and granule formation in Haloferax mediterranei. Appl Environ Microbiol, 2012, 78: 1946-1952

15 Lu Q H, Han J, Zhou L G, et al. Genetic and biochemical characterization of the poly(3-hydroxybutyrate-co-3-hydroxyvalerate) synthase in Haloferax mediterranei. J Bacteriol, 2008, 190: 4173-4180

16 Han J, Lu Q, Zhou L, et al. Molecular characterization of the phaE$C_{\mathrm{Hm}}$, genes, required for biosynthesis of poly(3-hydroxybutyr- ate) in the extremely halophilic Archaeon Haloarcula marismortui. Appl Environ Microb, 2007, 73: 6058-6065

17 Jendrossek D. Polyhydroxyalkanoate granules are complex subcellular organelles (carbonosomes). J Bacteriol, 2009, 191: 3195-3202

18 Baumeister W, Lembcke G. Structural features of archaebacterial cell envelopes. J Bioenerg Biomembr, 1992, 24: 567-575

19 Baumeister W, Wildhaber I, Phipps B M. Principles of organization in eubacterial and archaebacterial surface-proteins. Can J Microbiol, 1989, 35: 215-227

20 Houwink A L. Flagella, gas vacuoles and cell-wall structure in $\mathrm{Ha}$ lobacterium halobium: an electron microscope study. J Gen Microbiol, 1956, 15: 146-150

21 Koga Y, Morii H. Recent advances in structural research on ether lipids from archaea including comparative and physiological aspects. Biosci Biotech Bioch, 2005, 69: 2019-2034

22 Mescher M F, Hansen U, Strominger J L. Formation of lipid-linked sugar compounds in Halobacterium salinarium - presumed intermediates in glycoprotein synthesis. J Biol Chem, 1976, 251: 72897294

23 Wieland F, Lechner J, Bernhardt G, et al. Sulfation of a repetitive saccharide in halobacterial cell-wall glycoprotein occurrence of a sulfated lipid-linked precursor. Febs Lett, 1981, 132: 319-323

24 Wakai H, Nakamura S, Kawasaki H, et al. Cloning and sequencing 
of the gene encoding the cell surface glycoprotein of Haloarcula japonica strain TR-1. Extremophiles, 1997, 1: 29-35

25 Sumper M, Berg E, Mengele R, et al. Primary structure and glycosylation of the S-layer protein of Haloferax volcanii. J Bacteriol, 1990, 172: 7111-7118

26 Lechner J, Sumper M. The primary structure of a procaryotic glycoprotein. Cloning and sequencing of the cell surface glycoprotein gene of halobacteria. J Biol Chem, 1987, 262: 9724-9729

27 Fukuchi S, Yoshimune K, Wakayama M, et al. Unique amino acid composition of proteins in halophilic bacteria. J Mol Biol, 2003, 327: 347-357

28 Madern D, Ebel C, Zaccai G. Halophilic adaptation of enzymes. Extremophiles, 2000, 4: 91-98

29 Ng W V, Kennedy S P, Mahairas G G, et al. Genome sequence of Halobacterium species NRC-1. Proc Natl Acad Sci USA, 2000, 97: 12176-12181

30 Sleytr U B, Thorne K J. Chemical characterization of the regularly arranged surface layers of Clostridium thermosaccharolyticum and Clostridium thermohydrosulfuricum. J Bacteriol, 1976, 126: 377383

31 Yurist-Doutsch S, Chaban B, VanDyke D J, et al. Sweet to the extreme: protein glycosylation in Archaea. Mol Microbiol, 2008, 68: 1079-1084

32 Abu-Qarn M, Yurist-Doutsch S, Giordano A, et al. Haloferax volcanii AglB and AglD are involved in N-glycosylation of the S-layer glycoprotein and proper assembly of the surface layer. J Mol Biol, 2007, 374: 1224-1236

33 Abu-Qarn M, Giordano A, Battaglia F, et al. Identification of AglE, a second glycosyltransferase involved in $\mathrm{N}$ glycosylation of the Haloferax volcanii S-layer glycoprotein. J Bacteriol, 2008, 190: 3140-3146

34 Plavner N, Eichler J. Defining the topology of the N-glycosylation pathway in the halophilic archaeon Haloferax volcanii. J Bacteriol, 2008, 190: 8045-8052

35 Yurist-Doutsch S, Abu-Qarn M, Battaglia F, et al. aglF, aglG and aglI, novel members of a gene island involved in the N-glycosylation of the Haloferax volcanii S-layer glycoprotein. Mol Microbiol, 2008, 69: 1234-1245

36 Eichler J. Facing extremes: archaeal surface-layer (glyco)proteins. Microbiology, 2003, 149: 3347-3351

37 Mengele R, Sumper M. Drastic differences in glycosylation of related S-layer glycoproteins from moderate and extreme halophiles. J Biol Chem, 1992, 267: 8182-8185

38 Le Dain A C, Saint N, Kloda A, et al. Mechanosensitive ion channels of the archaeon Haloferax volcanii. J Biol Chem, 1998, 273: 12116-12119

39 Häse C C, Ledain A C, Martinac B. Purification and functional reconstitution of the recombinant large mechanosensitive ion-channel (Mscl) of Escherichia coli. J Biol Chem, 1995, 270: 18329-18334

40 Higgins C F. ABC transporters: from microorganisms to man. Annu Rev Cell Biol, 1992, 8: 67-113

41 Albers S V, Koning S M, Konings W N, et al. Insights into ABC transport in archaea. J Bioenerg Biomembr, 2004, 36: 5-15

42 Wanner C, Soppa J. Genetic identification of three ABC transporters as essential elements for nitrate respiration in Haloferax volcanii. Genetics, 1999, 152: 1417-1428

43 Woodson J D, Reynolds A A, Escalante-Semerena J C. ABC transporter for corrinoids in Halobacterium sp strain NRC-1. J Bacteriol, 2005, 187: 5901-5909

44 Lee S J, Böhm A, Krug M, et al. The ABC of binding-proteindependent transport in archaea. Trends Microbiol, 2007, 15: 389397

45 Bolhuis H, Palm P, Wende A, et al. The genome of the square archaeon Haloquadratum walsbyi: life at the limits of water activity. BMC Genomics, 2006, 7: 169

46 Barabote R D, Saier M H Jr. Comparative genomic analyses of the bacterial phosphotransferase system. Microbiol Mol Biol Rev, 2005, 69: 608-634

47 Anderson I, Rodriguez J, Susanti D, et al. Genome sequence of
Thermofilum pendens reveals an exceptional loss of biosynthetic pathways without genome reduction. J Bacteriol, 2008, 190: 29572965

48 Bolhuis A. Protein transport in the halophilic archaeon Halobacterium sp NRC-1: a major role for the twin-arginine translocation pathway? Microbiol-Sgm, 2002, 148: 3335-3346

49 Hutcheon G W, Bolhuis A. The archaeal twin-arginine translocation pathway. Biochem Soc T, 2003, 31: 686-689

50 Gimenez M I, Dilks K, Pöhlschroder M. Haloferax volcanii twin-arginine translocation substates include secreted soluble, C-terminally anchored and lipoproteins. Mol Microbiol, 2007, 66: 1597-1606

51 Berks B C, Palmer T, Sargent F. Protein targeting by the bacterial twin-arginine translocation (Tat) pathway. Curr Opin Microbiol, 2005, 8: 174-181

52 Rose R W, Brüser T, Kissinger J C, et al. Adaptation of protein secretion to extremely high-salt conditions by extensive use of the twin-arginine translocation pathway. Mol Microbiol, 2002, 45: 943-950

53 Widdick D A, Eijlander R T, van Dijl J M, et al. A facile reporter system for the experimental identification of twin-arginine translocation (Tat) signal peptides from all kingdoms of life. J Mol Biol, 2008, 375: 595-603

54 Berks B C, Palmer T, Sargent F. The Tat protein translocation pathway and its role in microbial physiology. Adv Microb Physiol, 2003, 47: $187-254$

55 Dilks K, Giménez M I, Pöhlschroder M. Genetic and biochemical analysis of the twin-arginine translocation pathway in halophilic archaea. J Bacteriol, 2005, 187: 8104-8113

56 Sargent F. The twin-arginine transport system: moving folded proteins across membranes. Biochem Soc Trans, 2007, 35: 835-847

57 Thomas $\mathrm{J}$ R, Bolhuis A. The tatC gene cluster is essential for viability in halophilic archaea. FEMS Microbiol Lett, 2006, 256: 44-49

58 Spudich J L, Yang C S, Jung K H, et al. Retinylidene proteins: structures and functions from archaea to humans. Annu Rev Cell Dev Biol, 2000, 16: 365-392

59 Spudich J L. Variations on a molecular switch: transport and sensory signalling by archaeal rhodopsins. Mol Microbiol, 1998, 28: 10511058

60 Oesterhelt D, Stoeckenius W. Rhodopsin-like protein from the purple membrane of Halobacterium halobium. Nat New Biol, 1971, 233: $149-152$

61 Henderson R, Unwin P N T. Three-dimensional model of purple membrane obtained by electron microscopy. Nature, 1975, 257: $28-32$

62 Subramaniam S, Hirai T, Henderson R. From structure to mechanism: electron crystallographic studies of bacteriorhodopsin. Philos T Roy Soc A, 2002, 360: 859-874

63 Khorana H G. Bacteriorhodopsin, a membrane protein that uses light to translocate protons. J Biol Chem, 1988, 263: 7439-7442

64 Krebs M P, Hauss T, Heyn M P, et al. Expression of the bacterioopsin gene in Halobacterium halobium using a multicopy plasmid. Proc Natl Acad Sci USA, 1991, 88: 859-863

65 Peck R F, Echavarri-Erasun C, Johnson E A, et al. brp and blh are required for synthesis of the retinal cofactor of bacteriorhodopsin in Halobacterium salinarum. J Biol Chem, 2001, 276: 5739-5744

66 Oesterhelt D, Stoeckenius W. Functions of a new photoreceptor membrane. Proc Natl Acad Sci USA, 1973, 70: 2853-2857

67 Stoeckenius W, Bogomolni R A. Bacteriorhodopsin and related pigments of halobacteria. Annu Rev Biochem, 1982, 51: 587-616

68 Jin Y D, Friedman N, Sheves M, et al. Bacteriorhodopsin (bR) as an electronic conduction medium: Current transport through bRcontaining monolayers. Proc Natl Acad Sci USA, 2006, 103: 8601-8606

69 Lanyi J K. X-ray crystallography of bacteriorhodopsin and its photointermediates: Insights into the mechanism of proton transport. Biochemistry(Moscow), 2001, 66: 1192-1196

70 Zhang F, Vierock J, Yizhar O, et al. The microbial opsin family of optogenetic tools. Cell, 2011, 147: 1446-1457 
71 Hirai T, Subramaniam S. Protein conformational changes in the bacteriorhodopsin photocycle: comparison of findings from electron and X-ray crystallographic analyses. PLoS ONE, 2009, 4: e5769

72 Lozier R H, Bogomolni R A, Stoeckenius W. Bacteriorhodopsin: a light-driven proton pump in Halobacterium halobium. Biophys $\mathrm{J}$, 1975, 15: 955-962

73 Krebs M P, Khorana H G. Mechanism of light-dependent proton translocation by bacteriorhodopsin. J Bacteriol, 1993, 175: 15551560

74 Dioumaev A K, Lanyi J K. Bacteriorhodopsin photocycle at cryogenic temperatures reveals distributed barriers of conformational substates. Proc Natl Acad Sci USA, 2007, 104: 9621-9626

75 Schobert B, Lanyi J K. Halorhodopsin is a light-driven chloride pump. J Biol Chem, 1982, 257: 306-313

76 Kolbe M, Besir H, Essen L O, et al. Structure of the light-driven chloride pump halorhodopsin at 1.8 angstrom resolution. Science, 2000, 288: 1390-1396

77 Hegemann P, Oesterhelt D, Steiner M. The photocycle of the chloride pump halorhodopsin I: azidecatalyzed deprotonation of the chromophore is a side reaction of photocycle intermediates inactivating the pump. EMBO J, 1985, 4: 2347-2350

78 Kunji E R S, von Gronau S, Oesterhelt D, et al. The three-dimensional structure of halorhodopsin to 5 angstrom by electron crystallography: A new unbending procedure for two-dimen- sional crystals by using a global reference structure. Proc Natl Acad Sci USA, 2000, 97: 4637-4642

79 Mukohata Y, Ihara K, Tamura T, et al. Halobacterial rhodopsins. J Biochem, 1999, 125: 649-657

80 Bogomolni R A, Spudich J L. Identification of a 3rd-rhodopsin-like pigment in phototactic Halobacterium halobium. Proc Natl Acad Sci-Biol, 1982, 79: 6250-6254

81 Hoff W D, Jung K H, Spudich J L. Molecular mechanism of photosignaling by archaeal sensory rhodopsins. Annu Rev Bioph Biom, 1997, 26: 223-258

82 Lutz I, Sieg A, Wegener A A, et al. Primary reactions of sensory rhodopsins. Proc Natl Acad Sci USA, 2001, 98: 962-967

83 Sasaki J, Spudich J L. Proton circulation during the photocycle of sensory rhodopsin II. Biophys J, 1999, 77: 2145-2152

84 Birge R R. Photophysics and molecular electronic applications of the rhodopsins. Annu Rev Phys Chem, 1990, 41: 683-733

85 Yan B, Nakanishi K, Spudich J L. Mechanism of activation of sensory rhodopsin I: evidence for a steric trigger. Proc Natl Acad Sci USA, 1991, 88: 9412-9416

86 Bergo V B, Spudich E N, Rothschild K J, et al. Photoactivation perturbs the membrane-embedded contacts between sensory rhodopsin II and its transducer. J Biol Chem, 2005, 280: 28365-28369

87 Shively J M, Cannon G C, Heinhorst S, et al. Bacterial and Archaeal Inclusions. In: Encyclopedia of Life Sciences. Chichester: John Wiley \& Sons, 2011

88 Offner S, Hofacker A, Wanner G, et al. Eight of fourteen gvp genes are sufficient for formation of gas vesicles in halophilic archaea. J Bacteriol, 2000, 182: 4328-4336

89 DasSarma S, Arora P, Lin F, et al. Wild-type gas vesicle formation requires at least ten genes in the gvp gene cluster of Halobacterium halobium plasmid pNRC100. J Bacteriol, 1994, 176: 7646-7652

90 Sivertsen A C, Bayro M J, Belenky M, et al. Solid-state NMR characterization of gas vesicle structure. Biophys J, 2010, 99: 1932-1939

91 Strunk T, Hamacher K, Hoffgaard F, et al. Structural model of the gas vesicle protein GvpA and analysis of GvpA mutants in vivo. Mol Microbiol, 2011, 81: 56-68

92 Hayes P K, Buchholz B, Walsby A E. Gas vesicles are strengthened by the outer-surface protein, GvpC. Arch Microbiol, 1992, 157: 229-234

93 Halladay J T, Jones J G, Lin F, et al. The rightward gas vesicle operon in Halobacterium plasmid pNRC100: identification of the gvpA and $g v p C$ gene products by use of antibody probes and genetic analysis of the region downstream of $g v p C$. J Bacteriol, 1993, 175: 684 692

94 Englert C, Pfeifer F. Analysis of gas vesicle gene expression in $\mathrm{Hal}$ oferax mediterranei reveals that GvpA and GvpC are both gas vesi- cle structural proteins. J Biol Chem, 1993, 268: 9329-9336

95 Offner S, Wanner G, Pfeifer F. Functional studies of the gvpACNO operon of Halobacterium salinarium reveal that the GvpC protein shapes gas vesicles. J Bacteriol, 1996, 178: 2071-2078

96 Walsby A E. Gas vesicles. Microbiol Rev, 1994, 58: 94-144

97 Shukla H D, DasSarma S. Complexity of gas vesicle biogenesis in Halobacterium sp. strain NRC-1: identification of five new proteins. J Bacteriol, 2004, 186: 3182-3186

98 Chu L J, Chen M C, Setter J, et al. New structural proteins of Halobacterium salinarum gas vesicle revealed by comparative proteomics analysis. J Proteome Res, 2011, 10: 1170-1178

99 Hezayen F F, Gutiérrez M C, Steinbüchel A, et al. Halopiger aswanensis sp. nov., a polymer-producing and extremely halophilic archaeon isolated from hypersaline soil. Int J Syst Evol Microbiol, 2010, 60: 633-637

100 Lillo J G, Rodriguez-Valera F. Effects of culture conditions on poly(beta-hydroxybutyric acid) production by Haloferax mediterranei. Appl Environ Microbiol, 1990, 56: 2517-2521

101 Hezayen F F, Rehm B H A, Eberhardt R, et al. Polymer production by two newly isolated extremely halophilic archaea: application of a novel corrosion-resistant bioreactor. Appl Microbiol Biot, 2000, 54: 319-325

102 Rehm B H. Polyester synthases: natural catalysts for plastics. Biochem J, 2003, 376: 15-33

103 Pötter M, Madkour M H, Mayer F, et al. Regulation of phasin expression and polyhydroxyalkanoate (PHA) granule formation in Ralstonia eutropha H16. Microbiology, 2002, 148: 2413-2426

104 Pötter M, Steinbüchel A. Poly(3-hydroxybutyrate) granule-associated proteins: impacts on poly(3-hydroxybutyrate) synthesis and degradation. Biomacromolecules, 2005, 6: 552-560

105 York G M, Stubbe J, Sinskey A J. The Ralstonia eutropha PhaR protein couples synthesis of the PhaP phasin to the presence of polyhydroxybutyrate in cells and promotes polyhydroxybutyrate production. J Bacteriol, 2002, 184: 59-66

106 DasSarma P, Coker J A, Huse V, et al. Encyclopedia of Industrial Biotechnology: Bioprocess, Bioseparation, and Cell Technology. Chichester: John Wiley \& Sons, 2010. 1-43

107 Sleytr U B, Huber C, Ilk N, et al. S-layers as a tool kit for nanobiotechnological applications. FEMS Microbiol Lett, 2007, 267: 131144

108 Sleytr U B, Egelseer E M, Ilk N, et al. S-Layers as a basic building block in a molecular construction kit. FEBS J, 2007, 274: 323-334

109 Sleytr U B, Sára M. Bacterial and archaeal S-layer proteins: structure-function relationships and their biotechnological applications. Trends Biotechnol, 1997, 15: 20-26

110 Schäffer C, Messner P. Surface-layer glycoproteins: an example for the diversity of bacterial glycosylation with promising impacts on nanobiotechnology. Glycobiology, 2004, 14: 31R-42R

111 Schuster B, Sleytr U B. Composite S-layer lipid structures. J Struct Biol, 2009, 168: 207-216

112 Birge R R, Gillespie N B, Izaguirre E W, et al. Biomolecular electronics: Protein-based associative processors and volumetric memories. J Phys Chem B, 1999, 103: 10746-10766

113 Stuart J A, Marcy D L, Wise K J, et al. Volumetric optical memory based on bacteriorhodopsin. Synthetic Met, 2002, 127: 3-15

114 Li Q, Stuart J A, Birge R R, et al. Photoelectric response of polarization sensitive bacteriorhodopsin films. Biosens Bioelectron, 2004, 19: 869-874

115 Birge R R. Protein-based computers. Sci Am, 1995, 272: 90-95

116 Bodo C. Sensory systems: Back into the light. Nat Rev Neurosci, 2010, 11, doi: 10.1038/nrn2893

117 Busskamp V, Duebel J, Balya D, et al. Genetic reactivation of cone photoreceptors restores visual responses in retinitis pigmentosa. Science, 2010, 329: 413-417

118 Cepko C. Neuroscience. Seeing the light of day. Science, 2010, 329: 403-404

119 Walsby A E, Hayes P K. Gas vesicle proteins. Biochem J, 1989, 264: 313-322

120 Stuart E S, Morshed F, Sremac M, et al. Antigen presentation using 
novel particulate organelles from halophilic archaea. J Biotechnol, 2001, 88: 119-128

121 Stuart E S, Morshed F, Sremac M, et al. Cassette-based presentation of SIV epitopes with recombinant gas vesicles from halophilic archaea. J Biotechnol, 2004, 114: 225-237

122 Sremac M, Stuart E S. Recombinant gas vesicles from Halobacterium $s p$. displaying SIV peptides demonstrate biotechnology potential as a pathogen peptide delivery vehicle. BMC Biotechnol, 2008, 8: 9

123 Chen G Q, Wu Q. The application of polyhydroxyalkanoates as tissue engineering materials. Biomaterials, 2005, 26: 6565-6578

124 Misra S K, Valappil S P, Roy I, et al. Polyhydroxyalkanoate (PHA)/inorganic phase composites for tissue engineering applications. Biomacromolecules, 2006, 7: 2249-2258

125 Sodian R, Sperling J S, Martin D P, et al. Fabrication of a trileaflet heart valve scaffold from a polyhydroxyalkanoate biopolyester for use in tissue engineering. Tissue Eng, 2000, 6: 183-188

126 Williams S F, Martin D P, Horowitz D M, et al. PHA applications: Addressing the price performance issue I. Tissue engineering. Int $\mathrm{J}$ Biol Macromol, 1999, 25: 111-121

127 Peters V, Rehm B H A. In vivo enzyme immobilization by use of engineered polyhydroxyalkanoate synthase. Appl Environ Microb, 2006, 72: 1777-1783

128 Brockelbank J A, Peters V, Rehm B H A. Recombinant Escherichia coli strain produces a ZZ domain displaying biopolyester granules suitable for immunoglobulin G purification. Appl Environ Microb, 2006, 72: 7394-7397

129 Grage K, Rehm B H A. In vivo production of scFv-Displaying biopolymer beads using a self-assembly-promoting fusion partner. Bioconjugate Chem, 2008, 19: 254-262

130 Peters V, Rehm B H A. Protein engineering of streptavidin for in vivo assembly of streptavidin beads. J Biotechnol, 2008, 134: 266274

131 Jahns A C, Haverkamp R G, Rehm B H A. Multifunctional inorganic-binding beads self-assembled inside engineered bacteria. Bioconjugate Chem, 2008, 19: 2072-2080

132 Banki M R, Wood D W. Inteins and affinity resin substitutes for protein purification and scale up. Microb Cell Fact, 2005, 4: 32

133 Anderson A J, Dawes E A. Occurrence, metabolism, metabolic role, and industrial uses of bacterial polyhydroxyalkanoates. Microbiol Rev, 1990, 54: 450-472

134 Lee S J, Park J P, Park T J, et al. Selective immobilization of fusion proteins on poly(hydroxyalkanoate) microbeads. Anal Chem, 2005, 77: 5755-5759

135 Banki M R, Gerngross T U, Wood D W. Novel and economical purification of recombinant proteins: intein-mediated protein purification using in vivo polyhydroxybutyrate (PHB) matrix association. Protein Sci, 2005, 14: 1387-1395

Open Access This article is distributed under the terms of the Creative Commons Attribution License which permits any use, distribution, and reproduction in any medium, provided the original author(s) and source are credited. 